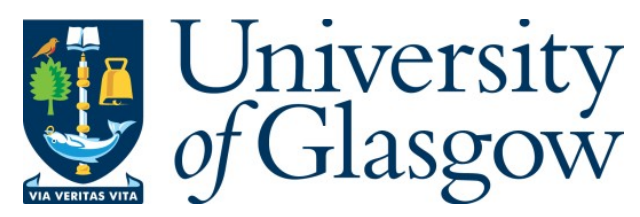

Wang, Y., Feng, G., Wang, J., Wei, F., Sun, Y. and Qin, S. (2021) Self-healing of Radio Access Network Slices. In: 2021 IEEE International Conference on Communications (ICC 2021), 14-23 Jun 2021, ISBN 9781728171227

(doi:10.1109/ICC42927.2021.9500408)

This is the Author Accepted Manuscript.

There may be differences between this version and the published version. You are advised to consult the publisher's version if you wish to cite from it.

http://eprints.gla.ac.uk/258363/

Deposited on: 10 November 2021

Enlighten - Research publications by members of the University of Glasgow http://eprints.gla.ac.uk 


\title{
Self-healing of Radio Access Network Slices
}

\author{
Yatong Wang*, Gang Feng*, Senior Member, IEEE, Jian Wang*, Fengsheng Wei*, Yao Sun ${ }^{\dagger}$, Shuang Qin* \\ *National Key Lab. on Communications, and Yangtze Delta Region Institute (Huzhou), \\ University of Electronic Science and Technology of China, Chengdu, China \\ ${ }^{\dagger}$ James Watt School of Engineering, University of Glasgow, Scotland, UK.S \\ E-mail:fenggang@uestc.edu.cn
}

\begin{abstract}
Radio Access Network (RAN) slicing is a promising architectural technology to address extremely diversified service demands for future mobile networks. As an essential requirement for RAN slicing, self-healing is to provide services with certain quality requirements by minimizing the impact of mobile network failings. In this paper, we propose a Multi-objective Pareto Optimization based Self-healing (MPOS) scheme to solve the SRANS problem. We model the SRANS problem as a multiobjective optimization problem with aim of maximizing the self-healing profits of individual RAN slices and demonstrate the NP-hardness. In proposed MPOS scheme, we employ selfconditioned GANs to replace the offspring reproduction module in the traditional Multi-Objective Evolutionary Algorithm (MOEA), where the insufficiency of diversity maintenance in MOEA is effectively overcome. Furthermore, we theoretically prove that MPOS framework is guaranteed to converge to the optimal Pareto solution set with probability 1 . Numerical results demonstrate that our MPOS scheme is effective in reducing the inverted generational distance of optimal Pareto solutions and achieving high profit and isolation level of RAN slices.
\end{abstract}

\section{INTRODUCTION}

In the era of $5 \mathrm{G}$ and beyond, mobile cellular networks are ultra-densely deployed by introducing a large number of lowcost Small Base Stations (SBSs) to cope with the explosion of data traffic demand. Unfortunately, the increasing number of Base Stations (BSs) in mobile wireless networks results in a higher failings ratio of access nodes [1]. Moreover, mobile network operators spend as much as $23 \%$ to $26 \%$ of their total revenue on maintaining and managing the networks [2]. Traditionally, mobile network operators have relied on manual experience to maintain, detect, diagnose, and recover their networks from any faults, which can be rather costly and inefficient. Therefore, it is inevitable for the telecom operators to solve this challenging issue for keeping revenue margins up. Self-healing as a key topic of self-organizing networks is to maintain the service continuity by minimizing the impact on performance (i.e. ensuring the quality of service (QoS) of users, reducing signaling overhead and the outage ratio) of the mobile network when network failings occur.

Most existing self-healing techniques include coverage area optimization, Signal-to-Interference-plus-Noise-Ratio (SINR) optimization, cell capacity optimization, spectral efficiency optimization [3] for outage compensation. However, self-healing

\footnotetext{
This work was supported by the National Key Research and Development Program of China under Grant number 2020YFB1806804, the National Science Foundation of China under Grant number 62071091 and ZTE IndustryAcademia-Research Cooperation Funds.
}

in burgeoning sliced RAN that provides customized services is vastly different from that in traditional RAN and has been rarely attended. RAN slices are constructed by Mobile Network Operators (MNOs) by renting a part of the resources from the Infrastructure Providers (IPs) to provide customized services [4]. When the network fails (e.g., SBSs malfunction), the deployed radio access network slices can be severely affected or even corrupt. Therefore, self-healing of RAN slice becomes indispensable.

Self-healing of RAN Slices (SRANS) means that mobile network operators repair their RAN slices to restore the function and ensure the QoS. Different from traditional resource allocation schemes in RAN slices that define the profits of RAN slices only with utility (i.e., service revenues) and deployment cost (i.e. the resources purchase cost paying to IPs), reconfiguration cost which is incurred in scheduling users also should be taken account in SRANS. In this paper, we model the SRANS problem as a Multi-objective Optimization Problem (MOP) and propose a Multi-objective Pareto Optimization based Self-healing (MPOS) scheme for RAN slices with aim of maximizing the self-healing profits of RAN slices while meeting transmission rate requirements. In MPOS scheme, in order to well define the profits of RAN slices, we propose the definition of RAN slice isolation level from interference perspective and the definition of reconfiguration cost. In MPOS, we design an opposition-based initialization strategy, and then construct a set of reference directions to ensure the diversity of solution set. For each iteration, a self-conditioned Generative Adversarial Network (GAN) is employed to cope with the weakness in the diversity of solution sets in evolutionary algorithms and generate an intermediate set of solutions. Based on this, objective normalization strategy and association are performed. Finally, we propose a novel fitness assignment technique for solution evaluation and selection. Numerical results demonstrate that our MPOS scheme is effective in reducing the Inverted Generational Distance (IGD) of optimal Pareto solutions and achieving high profits and isolation level of RAN slices.

\section{SySTEM MODELS}

\section{A. Sliced Heterogeneous RAN}

We consider a heterogeneous RAN scenario as depicted in Fig. 1, where MNOs construct their RAN slices by renting radio resources from IPs to provide customized services for 
their associated subscribers. In our model, the IPs have a set of Base Stations (BSs) $\mathcal{M}=\{1, \cdots, m, \cdots, M\}$ including a Marco Base Stations (MBS) and a set of SBSs $\{1, \cdots, M-1\}$ deployed in different areas of the network. Moreover, each BS has a certain coverage area $\rho_{m}, m \in \mathcal{M}$, where the coverage of the MBS covers all SBS areas. We assume that two BSs $m$ and $m^{\prime}$ are interfering with each other if $\rho_{m} \cap \rho_{m^{\prime}} \neq \emptyset$. A symmetric adjacency matrix $\boldsymbol{B}=\left(b_{m, m^{\prime}}\right), m, m^{\prime} \in \mathcal{M}$ is defined to represent the relationship between the two BSs such that $b_{m, m}=0$ for all $m \in \mathcal{M}, b_{m, m^{\prime}}=1$ if BSs $m$ and $m^{\prime}$ interfere with each other, and $b_{m, m^{\prime}}=0$ otherwise. We assume that spectrum resources are divided into resource blocks (RBs) [5], where each RB represents the minimum spatio-temporal scheduling unit. Moreover, we consider that all BSs share the same spectrum resources in order to improve the spectrum efficiency. Thus, the set of available resources at each BS is $\mathcal{R}=\{1, \cdots, r, \cdots, R\}$. The unit price of spectrum resources of BSs is denoted by $\operatorname{Pr}=\left[p r_{1}, \cdots, p r_{m}, \cdots, p r_{M}\right]$, which is determined by IPs.

In order to provide customized services to their subscribers, the MNOs construct a set of RAN slices denoted by $\mathcal{S}=$ $\{1, \cdots, s, \cdots, S\}$. Each RAN slice covers a specific set of Demand Points (DPs) [6] $\mathcal{D}^{s}=\left\{d_{1}^{s}, \cdots, d_{k}^{s}, \cdots, d_{K_{s}}^{s}\right\}$ with $K_{s}=\left|\mathcal{D}^{s}\right|$. The requested data rate of DP $d_{k}^{s}$ represents either the demand of single user node or accumulated demands from hotspots, which is denoted by $Q_{k, r e q}^{s}$. Let $\mathcal{M}_{k}^{s}$ be the set of BSs where DP $d_{k}^{s}$ is located in their coverage areas. Some of the radio spectrum resources of BSs are leased by MNOs to construct slices, while others are unused, as shown in Fig.1. Let $y_{s, k}^{m, r} \in\{0,1\}$ be the RB provisioning indicator such that $y_{s, k}^{m, r}=1$ if RB $r_{m} \in \mathcal{R}$ of SBS $m$ is leased to RAN slice $s$ for DP $d_{k}^{s}, y_{s, k}^{m, r}=0$ otherwise. Also, let $\boldsymbol{\pi}=\left(\boldsymbol{\pi}_{s}\right)_{s \in \mathcal{S}}$ be the slicing provisioning policy with $\boldsymbol{\pi}_{s}=\left(\boldsymbol{\pi}_{s, k}^{m}\right)_{k \in \mathcal{D}^{s}}^{s}, m \in \mathcal{M}_{k}^{s}$, where $\boldsymbol{\pi}_{s, k}^{m}$ represents the set of RBs on BS $m$ that are leased by RAN slice $s$ for DP $d_{k}^{s}$. Specifically, for any RB $r_{m} \in \mathcal{R}$ of BS $m$, we have that $r_{m} \in \boldsymbol{\pi}_{s, k}^{m} \Leftrightarrow y_{s, k}^{m, r}=1$.

However, when a failing occurs in an SBS, the fault supervision entity [7] will detect it. For example, if SBS 5 breaks down as shown in Fig. 1, self-healing needs to be conducted to recover Slice 2 and Slice 3 for ensuring their functions. Therefore, the set of affected RAN slices that need self-healing when SBS $m$ malfunctions is denoted by $\mathcal{S}^{*}=\left\{s \mid s \in \mathcal{S}, \boldsymbol{\pi}_{m}^{s} \neq \emptyset\right\}$, where we assume that only one SBS malfunctions at the same time. The self-healing scheme is to determine the new resource provisioning policy for affected RAN slices. Let $\boldsymbol{\pi}^{*}=\left(\boldsymbol{\pi}_{s}^{*}\right)_{s \in \mathcal{S}^{*}}$ denote the self-healing policy for RAN slicing. After self-healing of RAN slices, the achieved rate $Q_{k}^{s}$ of DP $d_{k}^{s}$ of RAN slice $s \in \mathcal{S}^{*}$ can be expressed as

$$
Q_{k}^{s}=\sum_{m} \sum_{r} y_{s, k}^{* m, r} B_{R B} \log _{2}\left(1+\frac{g_{s, k}^{m, r} p}{\sum_{m^{\prime} \in \mathcal{M}, m^{\prime} \neq \mathrm{m}} g_{s, k}^{m^{\prime}, r} p+\sigma^{2}}\right),
$$

where $y_{s, k}^{* m, k}$ indicates the RB provisioning indicator; $B_{R B}$ indicates the bandwidth of an RB; $g_{s, k}^{m, r}$ indicates the channel gain between $\mathrm{BS} m$ and $\mathrm{DP} d_{k}^{s}$ on RB $r$; and $p$ indicates the transmit power.

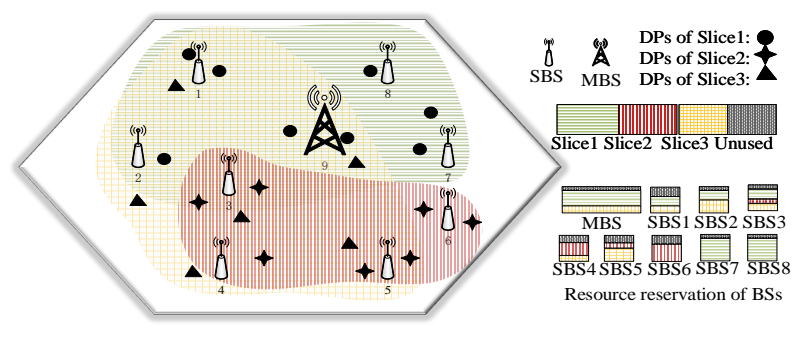

Fig. 1. An illustration of the Sliced Heterogeneous Radio Access Network

\section{B. Definition of Slicing Profit Function}

We define the slices profit, i.e., the revenue minus the deployment cost and reconfiguration cost. In the following, we define the revenue and cost respectively.

1) Revenue: One of the important features of network slicing is customization. Specifically, customization of a slice is to satisfy the service level agreements of subscribers by renting the resources from IPs [4]. Generally, the higher the service quality, the higher the profit of constructed RAN slices for MNOs. We define the revenue function in the aspect of customization of RAN slice $s$ as

$$
U_{s}^{c}=\sum_{k \in \mathcal{D}^{s}} \log \left(1+\frac{Q_{k}^{s}-Q_{k, r e q}^{s}}{Q_{k}^{s}}\right) \quad \forall s \in \mathcal{S}^{*},
$$

where (2) should be continuously increasing with service rate and strictly concave.

Moreover, isolation among slices is another fundamental feature and a crucial challenge of RAN slicing, which means that RAN slices should be operated in an isolated manner and stay unaffected by the traffic variations of other slices. Therefore, we proposed an effective method to improve the isolation among slices. The basic idea of the method is that the same RBs are assigned to the same slice when BSs are close enough to interfere among themselves, which is effective and efficient to mitigate the inter-slice interference and ensures the isolation of RAN slices.

\section{Definition 1. Isolation level of RAN slices from interference} perspective:

The isolation level $L_{s}$ of RAN slice $s$ is defined as $L_{s}=$ $\frac{\sum_{m} \sum_{m^{\prime} \in \mathcal{M}, m^{\prime} \neq m} \sum_{r} \sum_{k} y_{s, k}^{* m, r} y_{s, k}^{* m^{\prime}, r} b_{m, m^{\prime}}}{2 \sum_{m} \sum_{r} \sum_{k} y_{s, k}^{* n, r}}$, where the numerator of $L_{s}$ is the number of the identical rented RBs with mutually interfering BSs, and the nominator is twice the total number of rented $R B s$.

Similarly, MNOs make higher profit of RAN slice with higher isolation level for providing service quality. Thus, we define the revenue function from the perspective of isolation of RAN slice $s$ as

$$
U_{s}^{i}=\log \left(1+L_{s}\right) \quad \forall s \in \mathcal{S}^{*},
$$

where $U_{s}^{i}$ continuously increases with isolation level. Therefore, the revenue function of RAN slice $s$ is given by 


$$
U_{s}=\frac{U_{s}^{c}+U_{s}^{i}}{2} \quad \forall s \in \mathcal{S}^{*}
$$

2) Deployment Cost: MNOs deploy their RAN slices by renting certain physical resources (e.g., the spectrum resources of BSs) from IPs. Naturally, it incurs deployment cost, which depends on the amount and price of the rented resources. Therefore, the deployment cost $\phi_{s}$ of RAN slice $s$ is expressed as

$$
\phi_{s}=\frac{\sum_{m} \sum_{k} \sum_{r} y_{s, k}^{* m, r} p r_{m}}{P_{\text {total }}} \quad \forall s \in \mathcal{S}^{*},
$$

where $P_{\text {total }}=\sum_{i=1}^{M} R \operatorname{Pr}_{i}$.

3) Reconfiguration Cost: In contrast to the initial deployment of RAN slices, reconfiguration cost which is incurred in scheduling users also should be taken account in SRANS scheme design. An inappropriate SRANS solution can result in high signaling overhead and massive data migration. In view of this, the reconfiguration cost needs to follow the principle that the smaller change of the resource reservation policy, the lower of the reconfiguration cost. Hence, the reconfiguration cost function of RAN slice is defined as

$$
\vartheta_{s}=\frac{\sum_{m} \sum_{k}\left|\boldsymbol{\pi}_{s, k}^{* m}-\boldsymbol{\pi}_{s, k}^{* m} \cap \boldsymbol{\pi}_{s, k}^{m}\right|}{|\mathcal{R}||\mathcal{M}|} \quad \forall s \in \mathcal{S}^{*} .
$$

4) Total Profit of Slice: Considering the revenue, deployment cost, and reconfiguration cost, the total profit of slice $s$ under self-healing policy can be expressed as

$$
P_{s}=\alpha_{u} U_{s}-\alpha_{\phi} \phi_{s}-\alpha_{\vartheta} \vartheta_{s} \quad \forall s \in \mathcal{S}^{*},
$$

where the weights $\alpha_{u}, \alpha_{\phi}$, and $\alpha_{\vartheta}$ present the priority of 1), $2), 3)$. With SRANS, MNOs make a profit by providing and guaranteeing customized services for their subscribers.

\section{The PRoblems of SRANS}

The optimization goals are to maximize the self-healing profits of RAN slices. Problem SRANS is modeled as a MOP as follows:

$$
\begin{array}{lll}
\text { Max } & P=\left[P_{1}, \ldots, P_{s}, \ldots, P_{S}\right]^{T} & \\
\text { s.t. } & \sum_{s} \sum_{k} y_{s, k}^{* m, r} \leq 1 & \forall m, r \\
& Q_{k}^{s} \geq Q_{k, r e q}^{s} & \forall s, k \\
& y_{s, k}^{* m, r} \in\{0,1\} & \forall s, k, m, r
\end{array}
$$

where $P=\left[P_{1}, \ldots, P_{s}, \ldots, P_{S}\right]^{T}$ is the profit vector of RAN slices; Constraint (8.1) ensures the resource limitation. Constraint (8.2) states the minimum transmission rate requirement for DPs. Constraint (8.3) ensures that the RB provisioning indicators are binary variables.

In examining the NP-hardness of the SRANS problem, the following properties are exploited. 1) If a special case of a problem is NP-hard so is the general problem. 2) Proving the NP-hardness of the SOP entails the NP-hardness of the MOP.
Theorem 1. SRANS problem is NP-hard.

Proof: We first construct an instance of problem SRANS as follows. i) There exists one RAN slice in the heterogeneous RAN (i.e. $S=1$ ). ii) We set the weight $\alpha_{\phi}$ and $\alpha_{\vartheta}$ both to 0 . We refer the above instance of problem RANS to $\mathrm{P} 1$, which is an SOP under constraints (8.1) to (8.3). According to the aforementioned properties, we will show the NP-hardness of problem SRANS by a polynomial reduction from the Multi-dimensional Knapsack Problem (MKP) to P1. Then, we construct an MKB instance, where binary decision variable $x_{s, k}^{m, r}$ represents whether the item $(s, k, m, r)$ is selected or not. Moreover, there exist $\left(M R+\sum_{s} K_{s}\right)$ kinds of resource with constraint $C_{j}=\left\{\begin{array}{ll}1 & j<=M R \\ -Q_{k, r e q}^{s} & \text { otherwise }\end{array}\right.$. The item $(s, k, m, r)$ makes profit $p_{s, k}^{m, r}=-\alpha_{\phi} p r_{m}$ and consumes an amount $w_{s, k, m, r, j}=$ $\begin{cases}1 & j=m(R-1)+r \\ -B_{R B} \log _{2}\left(1+\frac{g_{s, k}^{m, r} p}{\sum_{m^{\prime} \in \mathcal{M}, m^{\prime} \neq \mathrm{m}} g_{s, k}^{m^{\prime}, r} p+\sigma^{2}}\right) & j>M R \\ 0 & \text { otherwise }\end{cases}$ from each resource $j$. The goal of MKP instance is to choose a subset of items with a maximum total profit. Selected items must, however, not exceed resource constraints expressed by the knapsack constraints. If there exists an optimal solution $\left\{x_{s, k}^{m, r}\right\}$ of MKP instance that maximizes the total profit, we can derive a solution of P1 from $\left\{x_{s, k}^{m, r}\right\}$ directly. On the other hand, if there is a solution of P1 defined by $\left\{y_{s, k}^{* m, r}\right\}$, we can find that $\left\{y_{s, k}^{* m, r}\right\}$ is therefore a solution of MKP instance. Thus, MKP has a solution if and only if P1 has a solution. In other words, MKP is reducible to P1. From the above analysis, the time complexity of the above reduction is polynomial. Since MKP is NP-hard, P1 and SRANS are NP-hard too. Thus, we cannot find the optimal solution to SRANS in polynomial time.

\section{Gan-based Multi-objective Pareto OPTIMIZATION ALGORITHM FOR SRANS}

\section{A. The Framework of the Proposed MPOS Scheme}

The basic framework of the proposed MPOS scheme is presented in Fig. 2. MPOS scheme starts with a novel oppositionbased initialization strategy, which effectively generates an initial set of feasible solutions to improve the convergence rate, and then construct a set of reference directions by dividing the objective space into several independent sub-regions to ensure the diversity of the solution set. For each iteration, MPOS scheme first employs self-conditioned GAN to generate an intermediate set of solutions, and performs objective strategy and association of each solution with the reference direction. Finally, solution evaluation and selection with a novel fitness assignment technique are performed to construct a set of solutions for converging to the Pareto front and keeping the diversity. In the following, we elaborate the implementation of MPOS scheme step by step. 


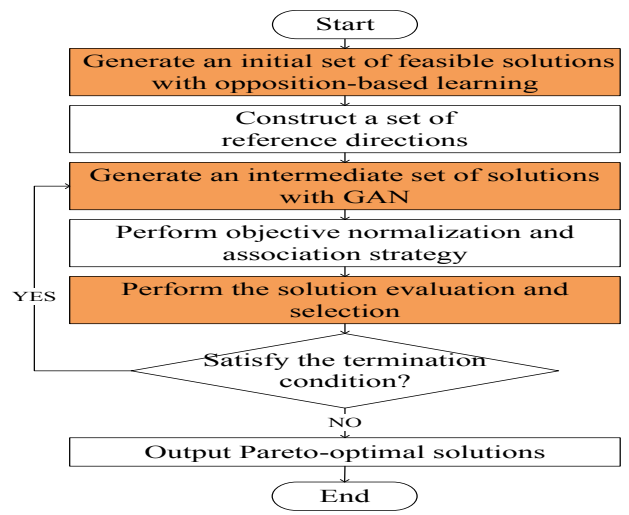

Fig. 2. Basic framework of the proposed MPOS scheme

\section{B. Opposition-based Initialization Strategy}

The initialization strategy affects the convergence rate of the algorithm. Recently a novel opposition-based learning method [8] has been proposed and proved effective in accelerating convergence. Inspired by this idea, we propose an oppositionbased initialization strategy, which can obtain more appropriate initialization candidate solutions without prior knowledge, so as to improve the efficiency of exploration. In the following, we first introduce the definition of opposition point in the decision space.

\section{Definition 2. Opposite Point:}

Let $X=\left[x_{1}, \cdots, x_{i}, \cdots, x_{N}\right]$ be a point of $N$-dimensional decision space, then its opposite point is defined as $\bar{X}=$ $\left[\overline{x_{1}}, \cdots, \overline{x_{i}}, \cdots, \overline{x_{N}}\right]$, where $\overline{x_{i}}=b_{i}-a_{i}-x_{i}$ with $x_{i} \in\left[a_{i}, b_{i}\right]$.

The proposed MPOS scheme randomly generates a group of initialized solutions, and compares the solutions with their opposition points, and then keep the better solutions as the initialized solution set.

\section{Reference Directions Construction}

Let us introduce the construction of reference directions below. Here we apply the widely used K-layer reference direction construction approach to generate the reference directions. First, we create the central reference direction and the extreme reference direction, which are respectively represented as $R C=(1 / S, \cdots, 1 / S)$ and $R E_{i}=\left(b_{1}, \cdots, b_{S}\right)$ where $b_{i}=1$ and $b_{j}=0$ for all $j \neq i, 1 \leq j \leq S, 1 \leq i \leq S$. The unit simplex can be partition into $S$ sub-simplexes, where each of sub-simplexes (denoted as Simp(i)) is bounded by points $R C, R E_{i}$, and $R E_{q}$ with $q=(i+1) \bmod S$. Then, a set of diverse reference directions $\mathcal{R D}_{i}=\left(\boldsymbol{D}_{i}^{k, t}\right)_{1 \leq k \leq K, 0 \leq t \leq k}$ for each Simp(i) is generated. The $k$ th layer of $t$ th reference direction $\boldsymbol{D}_{i}^{k, t}$ is represented as

$$
\boldsymbol{D}_{i}^{k, t}=R C+\frac{k}{K}\left(R E_{i}-R C\right)+\frac{t}{k+1} \frac{k}{K}\left(R E_{q}-R E_{i}\right),
$$

where $1 \leq k \leq K, 0 \leq t \leq k$, and $q=(i+1) \bmod S$. Note that $\boldsymbol{D}_{i}^{K, 0}$ is $R E_{i}$. Therefore, the set of all reference directions is expressed as $\mathcal{R D}=\mathcal{R} \mathcal{D}_{1} \cup \cdots \cup \mathcal{R} \mathcal{D}_{S} \cup\{R C\}$.

\section{GAN-based Exploration Strategy}

Recently, vigorously developed Generative Adversarial Networks (GANs) have great potential for applications in image synthesis, few-shot learning, and data augmentation. The GAN consists of two parts: generator network and discriminator network, as shown in Fig.3.

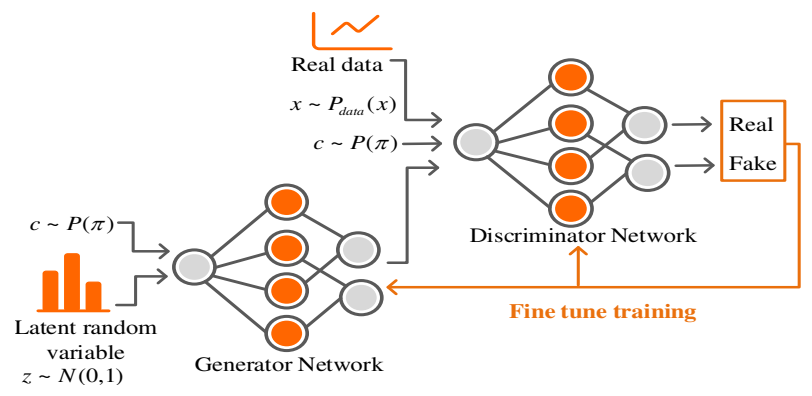

Fig. 3. An illustration of Generative Adversarial Networks

In this paper, we propose a self-conditioned GAN-based exploration strategy to solve SRANS problem by exploiting GANs' potential in generating an intermediate set of solutions effectively by leveraging their data augmentation capability. The generator network $G_{\theta}(\boldsymbol{z}, c)$ aims to generate potential solution set that looks similar to the real optimal solution cluster $c \sim P_{\pi}$ for $z \sim \mathcal{N}(0, I)$. The discriminator network $D_{\omega}(\boldsymbol{x}, c)$ is to distinguish between such generated potential solution and real optimal solution cluster. The generator and discriminator are trained to optimize the adversarial objective in the following minimax fashion:

$$
\begin{array}{r}
\min _{G} \max _{D} \mathcal{W}_{G A N}(G, D)=E_{c \sim P_{\pi}}\left[E_{x \sim P_{\text {data }}(x)}\left[\log \left(D_{\omega}(x, c)\right)\right]\right. \\
\left.\left.+E_{z \sim \mathcal{N}(0, I)}\left[D_{\omega}\left(G_{\theta}(z, c), c\right)\right)\right]\right] .
\end{array}
$$

The discriminator is inspired to a give low score for any solution that is not from cluster $c$, while the corresponding conditioned generator is penalized for generating solutions that are not from cluster $c$. Moreover, we employed $k$-means clustering to partition the solution set into $k$ clusters. Based on this, the proposed self-conditioned GAN-based exploration strategy can ensure the diversity of the generated solution without the mode collapse, which is one of the fundamental challenges for traditional GANs' training. We further employ the $\varepsilon$-greedy method to avoid stuck in local optimum, which is a pain point of traditional evolution algorithm. The basic idea is to generate intermediate solutions with probability $\varepsilon$ by using self-conditioned GAN exploration strategy and randomly generate intermediate solutions with probability $1-\varepsilon$.

\section{E. Objective normalization and solution association}

In order to map the objectives into a unit hypercube to achieve solution association, we first normalize the individual objectives of the solution set $\mathcal{E I}$ to form the normalization solution set $\overline{\mathcal{E}} \overline{\mathcal{I}}$. Then, the objectives of solution $e i \in \mathcal{E} \mathcal{I}$ are translated into:

$$
\overline{P_{s}}(e i)=\frac{P_{s}(e i)-P_{s}^{\min }}{P_{s}^{\max }-P_{s}^{\min }},
$$


where $s \in\{1, \cdots, S\}, P_{s}^{\min }=\min \left\{P_{s}(e j)\right\}, \forall e j \in \mathcal{E} \mathcal{I}$, $P_{s}^{\max }=\max \left\{P_{s}(e j)\right\}, \forall e j \in \mathcal{E} \mathcal{I}$ and $\overline{P_{s}}(e i)$ denotes normalization sth objectives of solution $e i$.

Next, we need to associate each solution in normalization set $\overline{\mathcal{E}} \overline{\mathcal{I}}$ with a reference direction. The associated reference direction $r d_{e i}$ of solution $e i$ is express as $r d_{e i}=\operatorname{argmin}\left(f\left(r d_{j}\right)\right)$, where $f\left(r d_{j}\right)=<\bar{P}(e i), r d_{j}>$ is the acute angle between vectors $\bar{P}(e i)=\left[\overline{P_{1}}(e i), \ldots, \overline{P_{s}}(e i), \ldots, \overline{P_{S}}(e i)\right]$ and $r d_{j} \in$ $\mathcal{R D}, j \in\{1, \ldots,|\mathcal{R D}|\}$.

\section{F. Solution evaluation and selection}

Fitness is an important concept to guide the expansion of solution set and environment selection. The fitness of a solution should conform to the following principle: the more number of other solutions dominated by the solution, the higher of the fitness value. Let $n_{e i}$ denote the number of other solutions dominated by solution $e i$, which is expressed by

$n_{e i}=\left\{\begin{array}{ll}-1 & \text { ei is invalid } \\ C(\{e j \mid e i \text { dominates } e j, \text { ej } \in \mathcal{E I}\}) & \text { otherwise }\end{array}\right.$,

where $C(\cdot)$ denotes the cardinality of a set.

In order to evaluate the solution more precisely and improve the diversity of the solution, we resort to the reference direction method. Therefore, when the number of solutions dominated by the solution $n_{e i}$ is the same, the smaller of acute angle between the solution and the associated reference direction, the higher of the fitness value. Let $f i t_{e i}$ denote the fitness value of the solution $e i$ : $f i t_{e i}=n_{e i}-\frac{\theta_{e i}}{\theta_{e i}+\theta}$, where $\theta_{e i}=<$ $\bar{P}(e i), r d_{e i}>$ and $\theta=\min \left\{<r d_{e i}, r d_{j}>_{j \neq i}\right\}$. Therefore, we select the best $N$ solutions of $\mathcal{E} \mathcal{I}$ according to the fitness value and include them into the set $\mathcal{I}$.

\section{G. Analysis of MPOS Scheme}

The proposed MPOS scheme for SRANS problem is $\mathrm{p}$ resented in Algorithm 1. Now, we analyze the property of convergence of MPOS scheme.

Lemma 1. In our MPOS scheme, the probability of any solution $f$ in the th iteration of intermediate solutions set $P(f \in \mathcal{E} \mathcal{I}(t))$ satisfies that $P(f \in \mathcal{E} \mathcal{I}(t)) \geq \sigma$ with $\sigma=\left(1-\varepsilon^{N}\right) / 2^{R M}$.

Proof: Let $f$ be any feasible solution of SRANS problem. Therefore, the probability of solution $f$ that is randomly generated in the intermediate solutions set $\mathcal{E} \mathcal{I}(t)$ is $\sigma=(1-$ $\left.\varepsilon^{N}\right) / 2^{R M}$, where $N, R$, and $M$ denote the size of the solution set, the number of RBs, and the number of BSs, respectively. Moreover, the probability that $f$ is selected in the intermediate solutions set $\mathcal{E} \mathcal{I}(t)$ is greater than the probability that $f$ is generated by random method in self-conditioned GAN-based exploration strategy. Therefore, we have $P(f \in \mathcal{E} \mathcal{I}(t)) \geq \sigma$.

Theorem 2. MPOS scheme is guaranteed to converge to the optimal Pareto solution set $\mathcal{F}^{*}$ with probability 1.
Proof: Let $f$ denote an arbitrary Pareto-optimal solution in optimal Pareto solution set $\mathcal{F}^{*}$. Once $f$ is searched by expanding population $\mathcal{E} \mathcal{I}(t)$, solution $f$ will always remain in $\mathcal{I}(t)$ until the end of iterations according to the solution evaluation and selection strategy. Therefore, we have $P(f \in$ $\mathcal{I}(t))=1-\prod_{i=1}^{t}(1-P(f \in \mathcal{E} \mathcal{I}(i))) \geq 1-(1-\sigma)^{t}$. Thus, $\lim _{t \rightarrow \infty} P(f \in \mathcal{I}(t)) \geq \lim _{t \rightarrow \infty} 1-(1-\sigma)^{t}=1$. when $t \rightarrow \infty, P(f \in \mathcal{I}(t)) \rightarrow 1$. At this point, the number of dominant solutions on set $\mathcal{I}(t)$ approaches 0 . Any nondominant solution will exist in the population with probability 1. As a result, MPOS scheme is guaranteed to converge to the optimal Pareto solution set $\mathcal{F}^{*}$ with probability 1 .

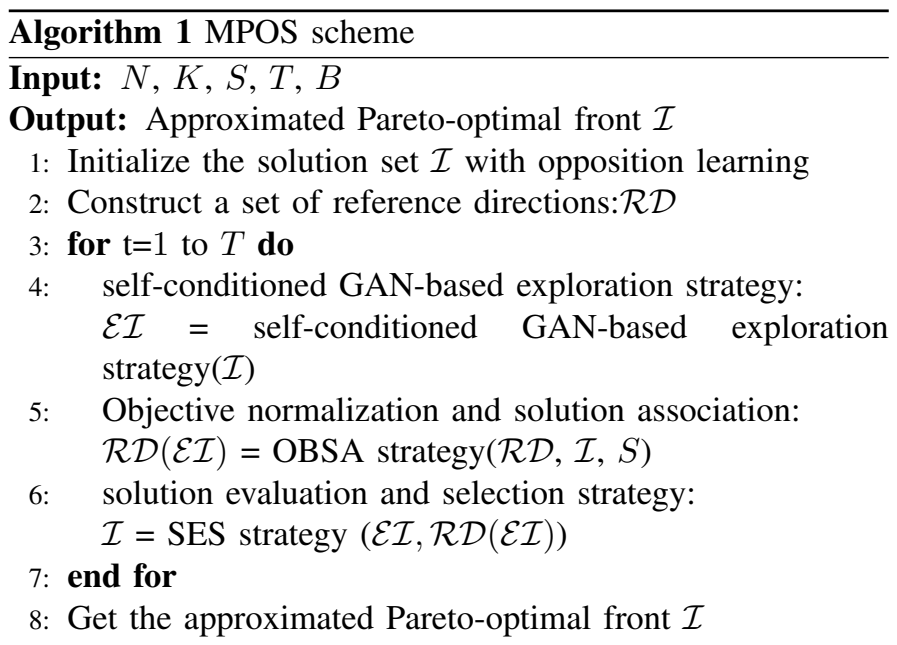

\section{Performance Evaluation}

We evaluate the performance of our proposed MPOS scheme by simulation in two typical urban environment named smallscale scenario and large-scale scenario. The service area of the small-scale scenario is $0.6 \mathrm{~km} \times 0.6 \mathrm{~km}$ with 6 deployed SBSs, one MBS, and 4 RAN slices, where 2 RAN slices are broken. The service area of the large-scale scenario is $1.0 \mathrm{~km} \times 1.0 \mathrm{~km}$ with 10 deployed SBSs, one MBS, and 7 RAN slices, where 3 RAN slices are broken. The other simulation parameters as shown in TABLE I. Moreover, we employ the widely used urban environment pathloss model:

$$
P L= \begin{cases}28.0+22 \log _{10}(d)+20 \log _{10}\left(f_{c}\right) & \text { MBS } \\ 32.4+21 \log _{10}(d)+20 \log _{10}\left(f_{c}\right) & \text { SBS. }\end{cases}
$$

To demonstrate the effectiveness of the proposed MPOS scheme, we compare it with Non Dominated Sorting Genetic Algorithm-III (NSGA-III) [9], which is an efficient algorithm for MOP.

Fig. 4 illustrates the Inverted Generational Distance (IGD) metric in small-scale scenario versus the number of iterations for these two algorithms with population number $N=100$ and $N=200$ respectively. IGD is used to evaluate the performance of the algorithm in terms of convergence rate and distribution diversity by calculating the sum of the minimum distance between each point on the real Pareto front(surface) and the 
TABLE I

SIMULATION PARAMETERS

\begin{tabular}{ll}
\hline Parameter & Value \\
\hline System Bandwidth & $20 \mathrm{MHz}$ \\
RB Bandwidth & $180 \mathrm{KHz}$ \\
Maximum SBS transmit power & $20 \mathrm{dBm}$ \\
Maximum mBS transmit power & $46 \mathrm{dBm}$ \\
Number of RB & 100 \\
Number of DPs per slice & $4 \sim 6$ \\
BSs antenna directivity & omni-directional \\
Thermal noise density & $-174 \mathrm{dBm} / \mathrm{Hz}$ \\
Carrier Frequency & $6 \mathrm{GHz}$ \\
Shadow fading & $4 \mathrm{~dB}$ \\
$\alpha_{u}, \alpha_{\phi}, \alpha_{\vartheta}$ & $1,1,1$ \\
\hline
\end{tabular}

set obtained by the algorithm, where the real Pareto front is evaluated by using violence search. Therefore, the smaller of IGD value, the better of overall performance of the algorithm. It can be observed that MPOS scheme with $N=200$ converges fastest, followed by MPOS scheme with $N=100$, NSGAIII with $N=200$ and NSGA-III with $N=100$ ranks last. Moreover, the IGD of MPOS scheme is lower than that of NSGA-III, since self-conditioned GAN and reference direction strategy are exploited to ensure the diversity of solutions.

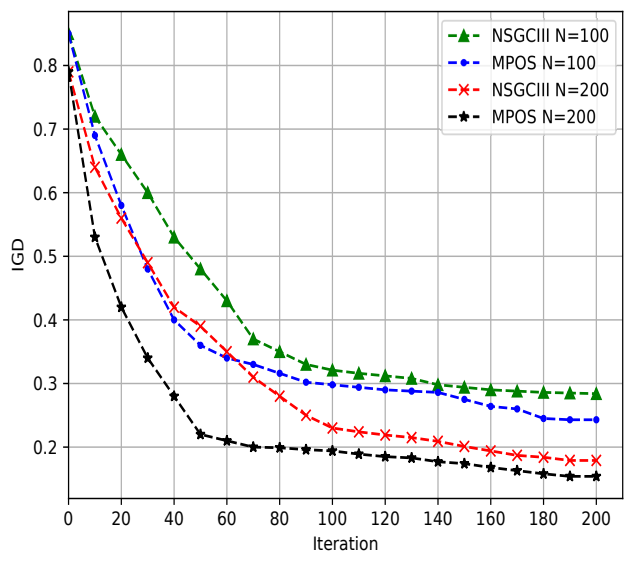

Fig. 4. The comparison of inverted generational distance metric in small-scale scenario vs. the number of iterations

We next compare the Average Isolation Level (AIL) of RAN slices in these two scenarios, as shown in Fig. 5. It can be observed that the AIL of RAN slices in MPOS with $N=200$ is higher than that in other three algorithms in smallscale scenario, followed by NSGA-III with $N=200$, MPOS with $N=100$, and NSGA-III with $N=100$ ranks last. This is because that our proposed MPOS scheme improves the diversity of the solution set to improve the exploration efficiency in approaching the optimal Pareto front. Due to the page limit, we do not give the results of large-scale scenario, whose trends are similar. However, the AIL of large-scale scenario is lower than that of small-scale scenario, due to the significant increase in the reuse level of resources.

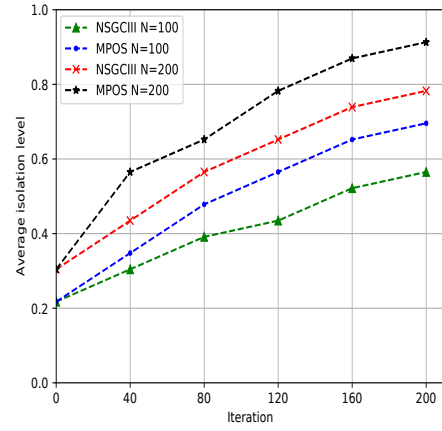

(a) Small-scale network

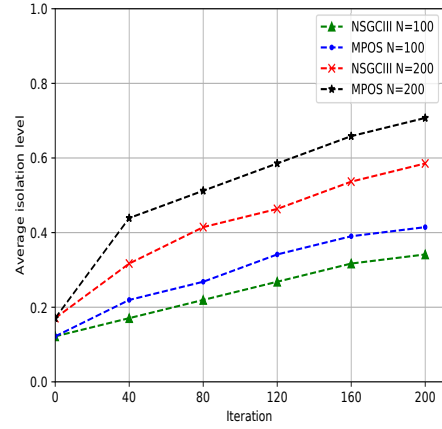

(b) Large-scale network
Fig. 5. The comparison of average isolation level of RAN slices with different scenario

\section{CONCLUSIONS}

In this paper, we have proposed an effective MPOS scheme for SRANS problem, which maximizes the self-healing profits of RAN slices while meeting transmission rate requirements. In SRANS scheme, we define RAN slice isolation level from the perspective of interference and defined the reconfiguration cost. Moreover, we employ self-conditioned GAN to cope with the weakness in the diversity of solution sets with evolutionary algorithms. Numerical results validate that our MPOS scheme is effective in reducing the IGD and achieving high profit and isolation level of RAN slices.

\section{REFERENCES}

[1] A. Asghar, H. Farooq, and A. Imran, "Self-healing in emerging cellular networks: review, challenges, and research directions," IEEE Communications Surveys \& Tutorials, vol. 20, no. 3, pp. 1682-1709, 2018.

[2] Aviat Networks, "Top ten pain points of operating networks," pp. 1-8, November 17, 2017. [Online]. Available: https://aviatnetworks.com/media/files/Top 10 Pain Points For Ops.pdf

[3] S. Fan and H. Tian, "Cooperative resource allocation for self-healing in small cell networks," IEEE Communications Letters, vol. 19, no. 7, pp. 1221-1224, 2015.

[4] Y. L. Lee, J. Loo, T. C. Chuah, and L. Wang, "Dynamic network slicing for multitenant heterogeneous cloud radio access networks," IEEE Transactions on Wireless Communications, vol. 17, no. 4, pp. 2146-2161, 2018.

[5] Y. Wang, G. Feng, Y. Sun, S. Qin, and Y. C. Liang, "Decentralized learning based indoor interference mitigation for 5g-and-beyond systems," IEEE Transactions on Vehicular Technology, vol. 69, no. 10, pp. 12 124-12 135, 2020.

[6] F. Bahlke, O. D. Ramos-Cantor, S. Henneberger, and M. Pesavento, "Optimized cell planning for network slicing in heterogeneous wireless communication networks," IEEE Communications Letters, vol. 22, no. 8, pp. 1676-1679, 2018.

[7] 3GPP, "Management and orchestration; Fault Supervision (FS) (Release 15)," 3rd Generation Partnership Project (3GPP), Technical Specification (TS) 28.545, 012018

[8] H. R. Tizhoosh, "Opposition-based learning: a new scheme for machine intelligence," International Conference on Computational Intelligence for Modelling, Control and Automation and International Conference on Intelligent Agents, Web Technologies and Internet Commerce (CIMCAIAWTIC'06), pp. 695-701, 2015.

[9] W. Mkaouer, M. Kessentini, A. Shaout, P. Koligheu, S. Bechikh, K. Deb, and A. Ouni, "Many-objective software remodularization using nsga-iii," ACM Transactions on Software Engineering and Methodology (TOSEM), vol. 24, no. 3, pp. 1-45, 2015. 